\title{
EFECTO DE LOS MANANO-OLIGOSACÁRIDOS SOBRE LOS PARÁMETROS PRODUCTIVOS DE CUYES (Cavia porcellus) DURANTE LA FASE DE ENGORDE
}

\section{Effect of Mannanoligosaccharides on the Productive Performance of Guinea Pigs (Cavia porcellus) during the Fattening Period}

\author{
Gonzalo Bazay D. ${ }^{1}$, Fernando Carcelén C. ${ }^{1,5}$, Miguel Ara G. ${ }^{1}$, \\ Ronald Jiménez A. ${ }^{4}$, Rosa González V. ${ }^{2}$, William Quevedo G. ${ }^{3}$
}

\section{Resumen}

\begin{abstract}
El estudio tuvo como objetivo evaluar el efecto de los manano-oligosacáridos (MOS) sobre la ganancia de peso vivo, consumo de alimento y conversión alimenticia de cuyes (Cavia porcellus) durante la fase de engorde. Se distribuyeron 75 cuyes machos de $28 \pm$ 3 días de edad en tres tratamientos aplicados como aditivos a una dieta basal: (a) Control (sin aditivo), (b) Antibiótico Promotor del Crecimiento (APC) (aditivo Zn-Bacitracina, 0.1 $\mathrm{g} / \mathrm{kg}$ ), y (c) MOS ((aditivo manano-oligosacárido, $0.5 \mathrm{~g} / \mathrm{kg}$ ). El periodo de aplicación de tratamientos y observaciones experimentales fue de seis semanas. No se detectaron efectos significativos de los tratamientos sobre ninguna de las variables evaluadas.
\end{abstract}

Palabras clave: cuy, prebiótico, manano-oligosacárido, ganancia de peso, consumo, índice de conversión alimenticia

\section{Abstract}

The present study aimed to evaluate the effect of mannan-oligosaccharides (MOS) on the body weight gain, feed intake and feed conversion rate of guinea pigs (Cavia porcellus) during the fattening period. Seventy five male guinea pigs ( $28 \pm 3$ days of age)

${ }^{1}$ Laboratorio de Bioquímica, Nutrición y Alimentación Animal, ${ }^{2}$ Laboratorio de Patología Aviar, ${ }^{3}$ Laboratorio de Zootecnia y Producción Agropecuaria, Facultad de Medicina Veterinaria, Universidad Nacional Mayor de San Marcos, Lima

${ }^{4}$ Centro de Investigación IVITA, El Mantaro, Universidad Nacional Mayor de San Marcos, Huancayo

${ }^{5}$ E-mail: nandodeme@gmail.com

Recibido: 17 de enero de 2013

Aceptado para publicación: 14 de febrero de 2014 
were randomly allocated in three experimental treatments applied as additives to a common basal diet as follows: (a) Control (no additive), (b) Growth Promoter Antibiotic (APC) (additive $\mathrm{Zn}$-Bacitracin $0.1 \mathrm{~g} / \mathrm{kg}$ ), and (c) MOS (additive mannan-oligosccharide, $0.5 \mathrm{~g} /$ $\mathrm{kg}$ ). The experimental period was six weeks. No significant differences between treatments and the control were observed.

Key words: guinea pigs, prebiotic, mannan-oligosaccharide, body weight gain, feed intake, feed conversion ratio

\section{INTRODUCCIÓN}

El cuy (Cavia porcellus) es una especie nativa de la zona andina del Perú, Ecuador, Colombia y Bolivia, adecuada para la crianza doméstica y comercial. Entre las bondades de la crianza de esta especie se puede mencionar la calidad de la carne, precocidad, rusticidad, prolificidad, bajos costos de producción y el precio de mercado. Por esto el cuy constituye un importante recurso alimenticio para la población, especialmente andina, así como fuente de ingresos económicos para el productor (Bustamante, 1993).

El auge de la crianza del cuy en los últimos años es debido al mayor consumo interno y a la creciente exportación de carne (MINAG, 2003). Este incremento en la demanda conlleva la necesidad de una crianza más intensiva y con mayor número de animales por área. Por otro lado, este nivel de intensificación acarrea problemas sanitarios que deben ser prevenidos (Chauca, 1995).

La inclusión de antibióticos en pequeñas dosis en la ración (niveles sub-terapéuticos) ha traído una mejora significativa en el desempeño de los animales de producción, proporcionando un aumento en la ganancia de peso, mejorando la conversión alimenticia y reduciendo la morbilidad y mortalidad (Gaskins et al., 2002; Yan y Gilbert, 2004). Estas ventajas han convertido al uso de APC en casi un estándar en el manejo de la alimentación y sanidad del cuy; sin embargo, puede ocasionarse un problema de salud públi- ca debido a la posibilidad de residuos de antibióticos en la carne y derivados del cuy, los cuales pueden generar resistencia a antibióticos en determinadas personas (Chauca, 1995). Este riesgo ha motivado la búsqueda de alternativas que mejoren el desempeño productivo de los animales en un grado similar al de los antibióticos promotores de crecimiento pero sin que presenten estas desventajas.

Una de estas alternativas son los prebióticos, sustancias indigestibles para los animales pero no para determinados microorganismos benéficos que tienen a su vez la propiedad de elevar el nivel de salud del hospedero (Torres, 1999). Dentro de los prebióticos con mayor potencial están los manano-oligosacáridos (MOS), tipo de sacáridos derivados de la pared celular de la levadura Saccharomyces cerevisiae, y uno de los microorganismos eucariotas más estudiados (Mewes et al., 1997). Los MOS actúan como un inhibidor competitivo en la colonización de la pared intestinal por parte de las bacterias patógenas y como estimulador del sistema inmune. La eficacia de los MOS ha sido demostrada en varias especies domésticas (Hooge, 2004; Mourãu et al., 2006; Bovera et al., 2010); sin embargo, se desconoce su comportamiento en el cuy, por lo que el objetivo del presente estudio fue determinar el efecto de los MOS sobre la ganancia de peso, consumo de alimento y conversión alimenticia de cuyes en engorde en sistemas de crianza semiintensiva en el valle del Mantaro, Perú. 


\section{Materiales y Métodos}

El estudio se realizó en la Estación Experimental El Mantaro del Instituto Veterinario de Investigaciones Tropicales y de Altura (IVITA), distrito de El Mantaro, departamento de Junín (3270 msnm, temperatura ambiental máxima de $19.9^{\circ} \mathrm{C}$, temperatura ambiental mínima de $-1.8^{\circ} \mathrm{C}$, precipitación media anual de $770 \mathrm{~mm}$ ). El periodo experimental fue de seis semanas (noviembre a diciembre de 2008).

Setentaicinco cuyes machos de las raza Inti, línea seleccionada por precocidad y prolificidad (Ministerio de Agricultura, s/f), de $28 \pm 3$ días de edad y peso promedio de $600 \mathrm{~g}$, fueron aleatoriamente distribuidos en 15 unidades experimentales consistentes en pozas con piso de cemento y paredes de madera y malla, con dimensiones de $1.66 \mathrm{~m}$ de largo, $1.39 \mathrm{~m}$ de ancho y $50 \mathrm{~cm}$ de altura. Las pozas fueron limpiadas, desinfectadas con cal, provistas con cama nueva y equipadas con dispensadoras de forraje y recipientes de arcilla de $0.5 \mathrm{~L}$ para el suministro de agua y concentrado. Las pozas, a su vez, fueron aleatoriamente distribuidas en tres tratamientos consistentes en aditivos mezclados con una dieta base: (a) Control (sin aditivo), (b) Antibiótico Promotor del Crecimiento (APC) (aditivo Zn-Bacitracina, $0.1 \mathrm{~g} / \mathrm{kg}$ ) y (c) MOS (aditivo manano-oligosacárido, $0.5 \mathrm{~g} / \mathrm{kg}$ ).

La dieta base consistió en $20 \%$ de concentrado (afrechillo de trigo) y $80 \%$ (materia seca) de forraje (rye grass + trébol) (Cuadro 1). El APC Zn-Bacitracina es comercializado bajo el nombre de Promozinb® (Laboratorios CUSA) y el MOS es un derivado de la pared celular de la levadura Saccharomyces cerevisae obtenido por secado por aspersión y comercializado con el nombre de Bio-Mos ${ }^{\circledR}$ (Alltech).

El concentrado y el forraje fueron ofrecidos una vez por día (08:00 y 10:00, respectivamente). El alimento rechazado (forraje y concentrado) se pesó semanalmente. Para obtener el porcentaje de materia seca, tanto del alimento ofrecido como del rechazado, se sometieron muestras a $60{ }^{\circ} \mathrm{C}$ en una estufa hasta peso constante.

Los animales se pesaron al inicio y fin del experimento, y la ganancia de peso vivo se obtuvo por diferencias de estos pesos. El consumo en materia seca se obtuvo de la diferencia entre la cantidad de alimento ofrecido y la cantidad de alimento rechazado, ambos expresados en materia seca. El índice de conversión alimenticia se calculó dividiendo el consumo total de materia seca entre la ganancia total de peso vivo. El pesado de los animales, alimento concentrado y forraje fueron realizados empleando una balanza electrónica con $1 \mathrm{~g}$ de sensibilidad.

El efecto de la adición de MOS o APC a la dieta basal, en términos de ganancia de peso vivo, consumo de alimento y conversión alimenticia, se estimó mediante un análisis de varianza correspondiente a un diseño completamente al azar con tres tratamientos y cinco repeticiones. Como prueba de uniformidad, se realizó un análisis de varianza antes de la aplicación de los tratamientos. El nivel de significación utilizado para todas las pruebas fue 0.05. Los análisis se ejecutaron con ayuda del paquete SAS/STAT® 9.2 (SAS Institute Inc., 2009).

\section{Resultados y Discusión}

El Cuadro 2 muestra las medias del consumo de materia seca, ganancia de peso y conversión alimenticia para cada uno de los tratamientos del experimento. En ningún caso se obtuvieron diferencias estadísticamente significativas con respecto al control y, en todos los casos, estas fueron de un orden inferior a $6 \%$, efecto marginalmente pequeño para propósitos prácticos (R. Jiménez, Huancayo, comunicación personal). Cabe señalar que la prueba preliminar de uniformidad del peso vivo no fue significativa, por lo que se podría atribuir la ausencia de diferen- 
Cuadro 1. Análisis proximal de los alimentos usados en el experimento

\begin{tabular}{lcc}
\hline Componente & Afrechillo & Trébol/ Rye grass \\
\hline Humedad, \% & 8.39 & 80.83 \\
Materia seca, \% & 91.61 & 19.17 \\
Proteína total, \% & 17.61 & 25.50 \\
Extracto etéreo, \% & 4.33 & 4.74 \\
Fibra cruda, \% & 10.12 & 21.2 \\
Ceniza, \% & 5.77 & 9.13 \\
Extracto libre de nitrógeno, \% & 62.17 & 39.4 \\
\hline
\end{tabular}

Cuadro 2. Efecto de la adición de un antibiótico promotor de crecimiento y del mananooligosacárido en el alimento sobre la ganancia de peso, consumo de materia seca y conversión alimenticia de cuyes de engorde en el valle de El Mantaro

\begin{tabular}{lcccc}
\hline \multirow{2}{*}{ Parámetros } & \multicolumn{4}{c}{ Tratamientos } \\
\cline { 2 - 5 } & Control & Antibiótico & MOS & Prob > F \\
\hline Consumo total de materia seca $(\mathrm{g})$ & 3101 & 3221 & 3289 & 0.502 \\
Ganancia de peso $(\mathrm{g})$ & 529 & 534 & 554 & 0.593 \\
Índice de Conversión Alimenticia & 5.84 & 6.04 & 5.94 & 0.668 \\
\hline
\end{tabular}

${ }^{1} p$-valor de la prueba de $F$

cias a un efecto de covarianza. Igualmente, es importante señalar que, tanto la conversión alimenticia, como la ganancia de peso obtenida en este estudio se compara favorablemente con aquellas obtenidas bajo condiciones similares (Jiménez y Huamán, 2010).

La ausencia de un efecto de la suplementación con MOS sobre la ganancia de peso ha sido encontrada en otros estudios realizados con conejos (Luick et al., 1992; Scapinello et al., 2001; Fonseca et al., 2004; Mourão et al., 2006; Volek et al., 2007); sin embargo, se encuentran algunas experiencias positivas a favor del MOS en conejos (Bersenyi y Gippert, 1995; Bovera et al., 2012).
Piccolo et al. (2010) encontraron un mayor consumo de materia seca en conejos por efecto del MOS; sin embargo, otros estudios demuestran que consumos similares en dietas suplementadas con MOS en comparación con los grupos control no suplementados (Mourão et al., 2006; Bovera et al., 2009, 2010), e incluso mayores consumos por efecto del APC (Bovera et al., 2009, 2010).

Los índices de conversión alimenticia fueron similares a otros estudios en conejos (Scapinello et al., 2001; Zanato et al., 2009; Piccolo et al., 2010). En contraste, Méndez et al. (1993), Aguilar et al. (1996) y Lebas (1996), trabajando con oligosacáridos de fructosa, encontraron una mejora en el ren- 
dimiento y conversión alimenticia de conejos. Asimismo, trabajos en aves suplementando MOS en la dieta presentaron mejoras significativas en el índice de conversión alimenticia (Hofacre et al., 2003; Hooge et al., 2003; Waldroup et al., 2003a,b; Sun et al., 2005).

Las diferencias en los resultados de este estudio con respecto a los encontrados en la literatura pueden estar vinculados a diferencias en la dosificación de los prebióticos, las condiciones ambientales, el estado fisiológico de los animales, las características de las dietas y, especialmente, en el nivel de desafío sanitario del estudio (Zanato et al., 2009).

Se ha reportado que los antibióticos (Sims et al., 2004) y aditivos (Hooge, 2004) son más efectivos bajo condiciones de enfermedad y estrés, tales como extremos de temperatura ambiental, hacinamiento y manejo deficiente, los cuales pueden ocurrir en la crianza intensiva comercial. El presente estudio se llevó a cabo en condiciones higiénicas adecuadas (óptimas instalaciones experimentales, medidas estrictas de bioseguridad, cama limpia, buena ventilación, y con una densidad media baja), lo que implica un desafío bacteriano mínimo. Bajo tales condiciones, los animales podrían no haber requerido de aditivos alimenticios para lograr una máxima respuesta productiva.

\section{Conclusiones}

La adición de MOS como de APC en la dieta de cuyes no tuvo un efecto significativo sobre los parámetros productivos del cuy en comparación con el grupo control.

\section{Literatura Citada}

1. Aguilar JC, Roca T, Sanz E. 1996. Fructo-oligosaccharides in rabbit diets. Study of efficiency in suckling and fattening periods. In: Proc VI World
Rabbit Congress. Tolouse, France. p 7377.

2. Bersenyi A, Gippert T. 1995. Effect of Bio-Mos supplementation upon the production traits of growing rabbits. In: I Egyptian Hungarian Poultry Conference. Alexandria, Eygpt. p 52-56.

3. Bovera F, Lestingi A, Marono S, Iannaccone F, Nizza S, Mallardo K, de Martino L, Tateo A. 2012. Effect of dietary mannan-oligosaccharides on in vivo performance, nutrient digestibility and caecal content characteristics of growing rabbits. J Anim Physiol Anim Nutr 96: 130-136.

4. Bovera F, Nizza S, Marono $S$, Mallardo K, Piccolo G, Tudisco R, De Martino L, et al. 2010. Effect of mannan oligosaccharides on rabbit performance, digestibility and rectal bacterial anaerobic populations during an episode of epizootic rabbit enteropathy. World Rabbit Sci 18(1): 9-16.

5. Bovera F, Marono S, Nizza S. 2009. Use of mannan oligosaccharides during «post-weaning enteric syndrome» in rabbits/ : effect on in vivo performance from 35 to 60 days. Ital J Anim Sci 8: 775-777.

6. Bustamante J. 1993. Producción de cuyes. Lima: Universidad Nacional Mayor de San Marcos. 259 p.

7. Chauca D. 1995. Fisiología digestiva. En: Serie Guía Didáctica: Crianza de cuyes. Lima: INIA. p 13-16.

8. Fonseca AP, Falcão L, Kocher A, Spring P. 2004. Effects of dietary mannan-oligosaccharide in comparison to oxytetracyclin on performance of growing rabbits. In: Proc VIII World Rabbit Congress. Puebla, Mexico. p 829833.

9. Gaskins HR, Collier CT, Anderson DB. 2002. Antibiotics as growth promotants: mode of action. Anim. Biotechnol 13: 29-42.

10. Hofacre CL, Bearcorn T, Collett S, Mathis G. 2003. Using competitive exclusion mannan-oligosaccharide and other intestinal products to control 
necrotic enteritis. J Appl Poultry Res 12: 60-64.

11. Hooge DM, Sims MD, Sefton AE, Connolly A, Spring P. 2003. Effect of dietary mannan oligosaccharide, with or without bacitracin or virginiamycin, on live performance of broiler chickens at relative high stocking density on new litter. J Appl Poultry Res 12: 461-467.

12. Hooge DM. 2004. Meta-analysis of broiler chicken pen trials evaluating dietary mannan oligosaccharide, 19932003. Int J Poultry Sci 3: 163-174.

13. Jiménez R, Huamán A. 2010. Manual para el manejo de reproductores híbridos especializados. Sub-Proyecto Desarrollo y Evaluación de Reproductores para Maximizar la Producción de Cuyes de Carne. INCAGRO-Instituto Veterinario de Investigaciones Tropicales y de Altura, FMV-UNMSM. Lima. 175 p.

14. Lebas F. 1996. Effects of fructooligosaccharides origin on rabbit's growth performance in 2 seasons In: Proc VI World Rabbit Congress, Tolouse, France. p 211-215.

15. Luick BR, El-Sayaad GAE, Cheeke PR. 1992. Effect of fructo-oligosaccharides and yeast culture on growth performance of rabbits. J Appl Rabbit Res 15: 1121-1128.

16. Méndez J, Mateos GG, Taboada S, Grobas S. 1993. Utilizacion de fructooligosaccharides (Profeed) em piensos de engorde de conejos. En: XVIII Simposio de Cunicultura. Granolers, España): Asociación Española de Cunicultura.

17. Mewes HW, Alberman $\mathrm{K}$, Bahr M, Frishmann D, Gleissner A, Hani J, Heumann K, et al. 1997. Overview of the yeast genome. Nature 387: 7-9.

18. Ministerio de Agricultura y Riego. Perú. s/f. Cuyes. [Internet], [1 diciembre 2013]. Disponible en http:// www.minag.gob.pe/portal/sector-agrario/pecuaria/situacion-de-las-actividades-de-crianza-y-produccion/ cuyes? start $=6$
19. Mourão JL, Pinheiro V, Alves A, Guedes CM, Pinto L, Saavedra MJ, Spring $P$, et al. 2006. Effect of mannan oligosaccharides on the performance, intestinal morphology and cecal fermentation of fattening rabbits. Anim Feed Sci Tech 126: 107-120.

20. Piccolo G, Bovera F, Meo CD. 2010. Mannan oligosaccharides as growth promoter in finishing rabbit: effect on in vivo performance and carcass traits. Ital J Anim Sci 8: 796-798.

21. SAS Institute Inc. 2009. SAS/STAT ${ }^{\circledR}$ 9.2 User's Guide, $2^{\text {nd }}$ ed. Cary, NC: SAS. $870 \mathrm{p}$.

22. Scapinello C, Faria HGD, Furlan AC, Michelan AC, Santolin ML da R. 2001. Efeito do uso de oligossacarideo manose e acidificantes em rações com alto teor de amido, para coelhos em crescimento. Acta Sci Maringa 23: 10391043.

23. Sims MD, Dawson KA, Newman KE, Spring P, Hooge DM. 2004. Effects of dietary mannnan oligosaccharide, bacitracin methylene disalicylate, or both on the live performance and intestinal microbiology of turkeys. Poultry Sci 83: 1148-1154.

24. Sun X, McElroy A, Webb KE, Sefton $A E$, Novak C. 2005. Broiler performance and intestinal alterations when fed drug-free diets. Poultry Sci 84: 12941302.

25. Torres R. 1999. Flora intestinal, probióticos y salud. Guadalajara, México: Ed Gráfica Nueva Yakult. 102 p.

26. Volek Z, Marounek M, Skrivanova V. 2007. Effect of a starter diet supplementation with mannan-oligosaccharide or inulin on health status, caecal metabolism, digestibility of nutrients and growth of early weaned rabbits. Animal 1:523-530.

27. Waldroup PW, Fritts CA, Yan F. 2003a. Utilization of Bio-Mos® mannan oligosaccharide and Bioplex ${ }^{\circledR}$ copper in broiler diets. Int J Poultry Sci 2: 44-52. 
28. Waldroup PW, Oviedo-Randon EO, Fritts CA. 2003b. Comparison of BioMos ${ }^{\circledR}$ and antibiotic feeding program in broiler diets containing copper sulphate. Int J Poultry Sci 2: 28-31.

29. Yan SS, Gilbert JM. 2004. Antimicrobial drug delivery in food animals and antimicrobial food safety concerns: an overview of in vitro and in vivo factors potentially affecting the animal gut microflora. Adv Drug Deliv Rev 56:1497-1521.

30. Zanato J, Lui J, Oliveira M, Junqueira $O$, Malheiros E, Scapinello C, Neto A. 2009. Effects of a probiotic and a prebiotic-containing diet on performance, carcass traits, and cecal and intestinal $\mathrm{pH}$ of growing rabbits. Biociências 17: 67-73. 